\title{
REPERCUSSÃO DO PROGRAMA BOLSA FAMÍLIA NA SEGURANÇA ALIMENTAR E NUTRICIONAL DAS FAMÍLIAS BENEFICIADAS DE UM MUNICÍPIO DO SUL CATARINENSE
}

DOI: http://dx.doi.org/10.18616/prat05

Carolina Kanarek da Silva UNESC - Universidade do Extremo Sul Catarinense carolinakanarek@gmail.com

Lara Canever UNESC - Universidade do Extremo Sul Catarinense

Rita Suselaine Vieira Ribeiro UNESC - Universidade do Extremo Sul Catarinense

Rosimar Fagundes Rodrigues UNESC - Secretaria de Assistência Social de Criciúma

Paula Rosane Vieira Guimarães UNESC - Universidade do Extremo Sul Catarinense paulag@unesc.net 


\section{INTRODUÇÃO}

Um marco na conquista pelo direito humano à alimentação adequada (DHAA) foi a introdução, na Constituição Federal de 1988, da alimentação como um direito social. O DHAA se relaciona intimamente com a segurança alimentar e nutricional (SAN), que visa promover a todos o acesso regular e permanente a alimentos de qualidade, em quantidade suficiente para suprir as necessidades nutricionais dos indivíduos (CUSTÓDIO; YUBA; CYRILLO, 2013).

No eixo que trata do acesso à alimentação, o DHAA é assegurado por meio de programas de alimentação e nutrição, de incentivos fiscais, de redução de tributos e de programas de transferência condicionada de renda, como o Programa Bolsa Família (PBF), que surgiu em 2003 como uma alternativa ao combate à fome e para promover a segurança alimentar e nutricional (MONTEIRO, 2014).

Para monitorar a percepção de segurança alimentar, insegurança e fome, usa-se como instrumento a Escala Brasileira de Insegurança Alimentar (EBIA), um questionário com 15 perguntas que foi validado em 2004 e que se mostra útil no auxílio de políticas de combate à fome (SEGALL-CORREA; MARIN-LEON, 2009).

No Brasil, o acesso aos alimentos é limitado, principalmente entre os grupos populacionais menos favorecidos financeiramente ou em situação de vulnerabilidade, como os beneficiários do PBF. A alimentação desses indivíduos pode apresentar-se comprometida, por isso é necessário averiguar se eles estão tendo suas necessidades nutricionais supridas e se têm acesso ao valor nutricional dos alimentos (MONTEIRO, 2014).

Apesar de muitas conquistas no campo da alimentação e da nutrição, o DHAA continua sendo violado, colocando a fome em um patamar de difícil superação. Vale ressaltar que a segurança alimentar é condicionada principalmente por fatores ligados à oferta e à demanda de alimentos na sociedade. Do lado da oferta, a segurança alimentar pressupõe a garantia de que haja disponibilidade suficiente e sustentável de alimentos que respeitem a cultura ali- 
mentar, que sejam fisicamente e economicamente acessíveis à população e que propiciem opções saudáveis de alimentação. Do lado da demanda, a segurança alimentar pressupõe a garantia de que todos os indivíduos saberão identificar e terão capacidade de adotar opções saudáveis de alimentação (CUSTÓDIO; YUBA; CYRILLO, 2013; BRASIL, 2004b).

A Coordenação Geral de Alimentação e Nutrição (CGAN) disponibiliza os formulários de marcadores de consumo alimentar no Sistema de Vigilância Alimentar e Nutricional (SISVAN Web) desde 2008, permitindo a avaliação do consumo alimentar da população brasileira. Os formulários visam identificar as práticas alimentares saudáveis e não saudáveis e propor a avaliação de alimentos consumidos no dia anterior, o que ameniza possíveis vieses de memória, ou seja, esquecimento em relação à alimentação realizada. Estão disponíveis três formulários: um para crianças menores de 6 meses, um para crianças de 6 a 23 meses e 29 dias de idade e outro para crianças com 2 anos de idade ou mais, adolescentes, adultos, idosos e gestantes. Desse modo, tais questionários podem ser aplicados a todos os integrantes da família. Em geral, o objetivo deste estudo foi verificar a situação de Insegurança Alimentar (IA) dessa população, bem como a qualidade da alimentação a que está tendo acesso.

Trata-se, portanto, de um tema relevante e atual, que se insere no papel da Universidade de incentivar o desenvolvimento regional por meio de ações que consolidem as políticas públicas de interesse da sociedade.

\section{MÉTODO}

Para avaliar a repercussão do Programa Bolsa Família na Segurança Alimentar e Nutricional das famílias beneficiadas em um município do Sul Catarinense o delineamento da pesquisa foi descritivo, de caráter quantitativo, transversal, com amostra não probabilística e amostragem por conveniência. Aceitaram participar da pesquisa 34 famílias de beneficiários do Programa Bolsa Família, totalizando 83 pessoas. A aprovação do Comitê de Ética em Pesquisa da UNESC, teve parecer n. 2.509.656. Para obtenção dos dados foram 
utilizados a Escala Brasileira de Insegurança Alimentar (EBIA) e os marcadores de consumo alimentar do Sistema de Vigilância Alimentar e Nutricional (SISVAN). A coleta dos dados ocorreu no Centro de Referência de Assistência Social (CRAS) com cronograma específico. Após concordar em participar da pesquisa e assinar o Termo de Consentimento Livre e Esclarecido, foram convidados a responder um questionário e ao procedimento antropométrico (pesar e medir). As variáveis antropométricas coletadas foram peso e estatura. Para a coleta do peso corporal, foi utilizada a balança do CRAS, com capacidade de $150 \mathrm{~kg}$, da marca Filizola e modelo plataforma. A estatura foi aferida em metros, com o auxílio de um antropômetro/régua vertical acoplado à balança de plataforma. Para crianças menores de dois anos, o peso e a altura foram coletados na carteirinha de vacinação.

\section{RESULTADOS}

A mulher é vista como o foco prioritário do PBF, tanto como titular quanto no cumprimento das condicionalidades impostas, sendo a principal interlocutora das ações de combate à pobreza. De acordo com o Ministério da Saúde (BRASIL, 2014a), essas condicionalidades representam a quebra do ciclo intergeracional da pobreza, por meio do acesso às políticas sociais, como educação, saúde e assistência social.

Segundo Brito e Costa (2015), a vulnerabilidade socioeconômica predomina nos domicílios monoparentais chefiados por indivíduos do sexo feminino, consequência das diferenças na forma de inserção e de valorização da mulher no mercado de trabalho, o que reflete em forma de menores rendimentos salariais. $\mathrm{O}$ PBF se consolidou como uma ferramenta de inclusão e promoção da autonomia das mulheres, que ao serem incluídas no programa são tomadas como representantes do grupo familiar, fortalecendo seus papéis na família e na comunidade (BRASIL, 2016).

Segundo o Instituto de Estudos Socioeconômicos (INESC, 2008), 94\% dos titulares do cartão do PBF são mulheres. Entre elas, 27\% são mães 
solteiras e $85 \%$ têm entre 15 e 49 anos. No caso da presente pesquisa, 97\% (33) das famílias pesquisadas foram representadas por titulares mulheres (mães), das quais 33 famílias foram representadas por 6\% (2) de titulares com menos de 20 anos de idade, confirmando assim o foco do programa.

Em pesquisa realizada no Rio Grande do Sul com as famílias beneficiárias do PBF de uma Unidade Básica de Saúde, a maior parte da população entrevistada era composta de mulheres (80\%) com idade entre 20 e 59 anos. A população de 2 a 10 anos correspondia a $28,75 \%$ e a de 10 a 19 anos a $16,25 \%$ (ROSA, 2011).

No estudo de Cabral et al. (2013), os quais avaliaram o estado nutricional, o consumo e a segurança alimentar de beneficiários do PBF residentes nas favelas de Maceió ( $\mathrm{AL})$, também na maioria das famílias a mãe era responsável pelo benefício $(89,2 \%)$. Das 321 crianças avaliadas, 50,2\% eram meninos e 49,8\% meninas. Os adultos estudados $(\mathrm{n}=326)$ tinham idade média de 34,3 anos ( $\mathrm{DP}=8,7$ anos), sendo $38,7 \%$ do sexo masculino e $61,3 \%$ do sexo feminino.

Neste estudo, das 83 pessoas que fizeram parte das 34 famílias entrevistadas, 59\% (49) pertencem ao gênero feminino e 40,1\% (34) ao gênero masculino. Percebem-se resultados semelhantes quanto aos percentuais, apesar dos números absolutos serem menores, visto que as crianças com menos de 10 anos - 64,7\% (11) - pertencem ao sexo masculino e 35,3\% (6) ao feminino. Os adultos têm uma média de idade de 30,6 anos $( \pm 8,8)$, o que corresponde a $75,6 \%$ (31) do sexo feminino e $24,4 \%$ (10) do sexo masculino.

Utilizando os marcadores do SISVAN (2015), foram coletados os dados a respeito do consumo alimentar das famílias por faixa etária. O SISVAN tem como objetivo realizar o diagnóstico descritivo e analítico da situação alimentar e nutricional da população brasileira, contribuindo para o conhecimento da natureza e magnitude dos problemas de nutrição, identificando também as áreas geográficas, segmentos sociais e grupos populacionais acometidos de maior risco aos agravos nutricionais (PEREZ et al., 2013).

Quanto ao consumo alimentar, a única criança menor de 6 meses, com 3 meses de vida, é do sexo masculino, estava em aleitamento materno 
Misto ou Parcial (quando a criança recebe outros tipos de leite além do materno). Não fazia uso de mingau, água, chás, mas usava fórmula para complementar sua alimentação (BRASIL, 2015b).

As crianças de 6 a 24 meses incompletos totalizaram 5, com idades entre 7 e 23 meses, uma média de 16 meses $( \pm 7,4)$, sendo $60 \%$ (3) do sexo feminino e $40 \%$ (2) do masculino. A tabela 2 mostra a frequência alimentar do consumo de leite materno, de fruta e de comida de sal em crianças de 6 a 24 meses incompletos que foi referida pelos pais e/ou responsáveis pela criança. Destas, apenas $20 \%$ (1) consumiram leite materno no dia anterior à pesquisa, $60 \%$ (3) comeram frutas e todas consumiram alguma comida de sal pelo menos uma vez ao dia. Segundo o Ministério da Saúde (BRASIL, 2015b), por ser da mesma espécie, o leite materno contém todos os nutrientes essenciais para o crescimento e desenvolvimento da criança, além de ser mais bem digerido quando comparado a outros leites. Ele é capaz de suprir sozinho as necessidades nutricionais das crianças nos primeiros seis meses de vida e continua sendo uma importante fonte de nutrientes até o segundo ano de vida.

Segundo as orientações para a avaliação dos marcadores de consumo alimentar para atenção básica (BRASIL, 2015a), o consumo de leite materno até os 2 anos é importante devido à proteção contra diversas doenças nos primeiros anos de vida. Seguir em aleitamento materno até os 2 anos ou mais estende a oferta de energia e de micronutrientes ao bebê, especialmente o ferro. Porém, faz-se necessário orientar as mães de que nesse período apenas o leite do peito não será suficiente para suprir as necessidades das crianças, sendo necessário complementá-lo com uma alimentação saudável e planejada adequadamente.

Os dois primeiros anos de vida da criança são caracterizados por crescimento acelerado e enormes aquisições no processo de desenvolvimento, incluindo habilidades para receber, mastigar e digerir outros alimentos, além do leite materno. O governo brasileiro e órgãos representativos no Brasil recomendam o aleitamento materno até os 6 meses de vida, e adequações de práticas de alimentação complementar a partir dessa idade (BRASIL, 2015b). 
A alimentação complementar é compreendida como aquela fornecida no período em que os outros alimentos ou líquidos são oferecidos em adição ao leite materno. Qualquer alimento ou líquido oferecido nesse período é chamado de alimento complementar, que, quando adequado, deve fornecer alimentos ricos em energia e micronutrientes, sem contaminação, sem excesso de sal ou de condimentos, evitando-se alimentos industrializados (DIAS; FREIRE; FRANCESCHINI, 2010).

Segundo as orientações para a avaliação dos marcadores de consumo alimentar para atenção básica (BRASIL, 2015a), os alimentos complementares devem ser oferecidos três vezes ao dia (papa salgada e de frutas), sendo fundamental oferecer duas frutas diferentes por dia e fontes de vitamina $\mathrm{C}$ após a refeição principal para aumentar a absorção do ferro.

A frequência alimentar das crianças de 6 a 24 meses incompletos revelou que $80 \%$ (4) consumiram algum leite que não o do peito, mingau com leite, algum tipo de legume, vegetais ou frutas de cor alaranjada; 60\% (3) consumiram iogurte, macarrão instantâneo, salgadinho de pacote ou biscoito salgado; $20 \%$ (1) consumiram verdura de folha, hambúrgueres ou embutidos; $100 \%$ (5) consumiram carne ou alguma fonte de carboidratos (arroz, aipim, macarrão, farinha ou batata), 40\% (2) consumiram algum tipo de doce ou guloseima e nenhuma criança consumiu fígado ou bebidas adoçadas.

Conforme Carvalho et al. (2015), as crianças são um grupo vulnerável devido ao crescimento rápido e à imaturidade fisiológica e imunológica. A nutrição deve ser adequada principalmente nesses primeiros anos de vida, para que o crescimento e desenvolvimento aconteçam de forma saudável. Alimentos ricos em gorduras saturadas e trans, açúcares e sódio podem comprometer o estado nutricional e levar ao desenvolvimento de carências ou excessos nutricionais.

No estudo de Coradi, Bottaro e Kirsten (2017), com amostra de 75 crianças de 6 meses a 2 anos de idade, sobre o consumo alimentar em Arvorezinha-RS, observou-se um grande consumo de frutas $(87,3 \%)$, comida de sal $(95,31 \%)$, legumes $(70,31 \%)$, carnes $(71,88 \%)$ e arroz, batata, inhame, 
mandioca, farinha, macarrão (90,4\%). Dessas crianças, 57,81\% ainda recebiam aleitamento materno e 27,41\% eram beneficiárias do PBF.

Toloni et al. (2011) analisaram a introdução de alimentos industrializados e de alimentos de uso tradicional da dieta de 270 crianças frequentadoras de berçários de oito creches públicas e filantrópicas do município de São Paulo. Os resultados mostram que para aproximadamente $2 / 3$ das crianças foram oferecidos, antes dos 12 meses, alimentos com potencial obesogênico, como macarrão instantâneo, salgadinhos, bolacha recheada, suco artificial, refrigerante e bala/pirulito/chocolate. $\mathrm{O}$ resultado foi semelhante, apesar da diferença amostral, visto que 60\% (3) consumiram iogurte, macarrão instantâneo, salgadinho de pacote ou biscoito salgado.

As crianças de 2 a 10 anos incompletos chegaram ao total de 11, com média de idade de 7,0 anos $( \pm 1,7)$, idade mínima de 3 anos e máxima de 9 anos. Destas, $72,7 \%$ (8) eram do sexo masculino e $27,3 \%$ (3) do sexo feminino. Quanto aos adolescentes entre 10 e 19 anos, a média de idade foi de 13,4 anos $( \pm 2,6)$, com $52 \%$ (13) do sexo masculino e $48 \%$ (12) feminino. A tabela 1 mostra as refeições realizadas ao longo do dia por essas crianças e adolescentes.

Tabela 1 - Refeições realizadas ao longo do dia por crianças de 2 a 10 anos incompletos, e adolescentes, beneficiários do Programa Bolsa Família de um município do sul catarinense.

\begin{tabular}{c|c|c|c|c}
\hline \multirow{2}{*}{ Refeições } & \multicolumn{2}{|c|}{$\begin{array}{c}\text { Crianças de 2 a 10 anos } \\
\text { incompletos (n=11) }\end{array}$} & \multicolumn{2}{c}{ Adolescentes (n=25) } \\
\cline { 2 - 5 } & Sim & Não & Sim & Não \\
\hline Café da manhã & $9(81,8 \%)$ & $2(18,2 \%)$ & $21(84 \%)$ & $4(16 \%)$ \\
\hline Lanche da manhã & $7(63,6 \%)$ & $4(36,4 \%)$ & $11(44 \%)$ & $14(56 \%)$ \\
\hline Almoço & $11(100 \%)$ & - & $21(84 \%)$ & $4(16 \%)$ \\
\hline Lanche da tarde & $9(81,8 \%)$ & $2(18,2 \%)$ & $22(88 \%)$ & $3(12 \%)$ \\
\hline Janta & $11(100 \%)$ & - & $25(100 \%)$ & - \\
\hline Ceia & $5(45,5 \%)$ & $6(54.5 \%)$ & $16(64 \%)$ & $9(36 \%)$ \\
\hline
\end{tabular}

Fonte: Dados da pesquisa, 2018. 
Nenhuma das crianças e nenhum dos adolescentes entrevistados fizeram as seis refeições diárias indicadas. O Guia Alimentar para População Brasileira (BRASIL, 2014a) recomenda que sejam ofertadas refeições complementares, além das três refeições principais (café da manhã, almoço e janta), para crianças e adolescentes em fase de crescimento, devido ao aumento da necessidade energética. Semelhante ao resultado da pesquisa, Barros et al. (2011), analisando o banco de dados de uma unidade de saúde com 20 crianças de um a cinco anos, em Campina Grande-PB, chegaram ao resultado de que $65 \%$ das crianças presentes na amostra realizavam de uma a três refeições diárias.

Também semelhante aos resultados obtidos na pesquisa, um estudo de avaliação do consumo alimentar de pré-escolares de Nova Rita-RS, com crianças de 2 a 5 anos mostrou que crianças atendidas em escolas de período integral realizavam ao menos 4 refeições ao dia, sendo elas desjejum, almoço, lanche da tarde e jantar. Com isso, pode-se observar o papel importante da escola nos hábitos alimentares infantis (ZVEIBRÜCKER; MIRAGLIA, 2012).

Em relação ao hábito das crianças e dos adolescentes de realizarem as refeições assistindo à TV, mexendo no celular ou no computador foi observado que $55 \%$ das crianças e $72 \%$ dos adolescentes, ou seja, mais da metade deste público possui este costume.

Conforme Fiates, Amboni e Teixeira (2008), a ocorrência de sobrepeso e obesidade em crianças e adolescentes está associada ao hábito de assistir à TV, o qual promove o sedentarismo, sendo associado também ao baixo condicionamento físico. Além disso, sabe-se que os anúncios de alimentos veiculados na TV têm o poder de efetivamente promover o seu consumo, influenciando diretamente nos hábitos alimentares das crianças.

Na pesquisa de Moreira e Carraschi (2017) sobre a avaliação do consumo alimentar de crianças menores de 5 anos, da amostra de 326 crianças, $48,2 \%$ (157) possuíam o hábito de realizar as refeições assistindo à TV ou mexendo no celular/computador.

Corrêa e Martins (2018) avaliaram o estado nutricional de 73 escolares de 7 a 12 anos de idade, obtendo como resultado o percentual de crianças 
com o hábito de realizarem as refeições assistindo à TV ou mexendo no celular/computador de 50,7\%.

A tabela a seguir mostra a distribuição dos marcadores do consumo alimentar do SISVAN por crianças de 2 a 10 anos incompletos e adolescentes. O consumo elevado de hambúrgueres/embutidos, de bebidas adoçadas, de macarrão instantâneo, de salgadinhos de pacote ou de biscoitos salgados, de biscoitos doces e de guloseimas por adolescentes é um fator preocupante.

Tabela 2 - Distribuição dos marcadores do consumo alimentar do SISVAN por crianças de 2 a 10 anos incompletos, e adolescentes, beneficiários do PBF de um município do sul catarinense

\begin{tabular}{|c|c|c|c|c|c|c|}
\hline \multirow{2}{*}{ Alimentos } & \multicolumn{3}{|c|}{$\begin{array}{l}\text { Crianças de } 2 \text { a } 10 \text { anos } \\
\text { incompletos }(n=11)\end{array}$} & \multicolumn{3}{|c|}{ Adolescentes $(n=25)$} \\
\hline & Sim & Não & Não Sabe & Sim & Não & $\begin{array}{l}\text { Não } \\
\text { Sabe }\end{array}$ \\
\hline Feijão & $7(63,6 \%)$ & $2(18,2 \%)$ & $2(18,2 \%)$ & $14(56 \%)$ & $4(16 \%)$ & $7(28 \%)$ \\
\hline $\begin{array}{c}\text { Frutas frescas (não } \\
\text { considerar suco } \\
\text { de frutas) }\end{array}$ & $3(27,3 \%)$ & $7(63,6 \%)$ & $1(9,1 \%)$ & $9(36 \%)$ & $9(36 \%)$ & $7(28 \%)$ \\
\hline $\begin{array}{l}\text { Verduras e/ou legumes } \\
\text { (não considerar batata, } \\
\text { mandio-ca, aipim, } \\
\text { macaxeira, cará } \\
\text { e inhame) }\end{array}$ & $3(27,3 \%)$ & $7(63,6 \%)$ & $1(9,1 \%)$ & $8(32 \%)$ & $10(40 \%)$ & $7(28 \%)$ \\
\hline $\begin{array}{l}\text { Hambúrguer e/ } \\
\text { ou embutidos } \\
\text { (presunto, mor- } \\
\text { tadela, salame, } \\
\text { linguiça, salsicha) }\end{array}$ & $1(9,1 \%)$ & $9(81,8 \%)$ & $1(9,1 \%)$ & $6(24 \%)$ & $12(48 \%)$ & $7(28 \%)$ \\
\hline $\begin{array}{c}\text { Macarrão instantâ- } \\
\text { neo, salgadinhos } \\
\text { de pacote ou } \\
\text { biscoitos salgados }\end{array}$ & $1(9,1 \%)$ & $8(72,7 \%)$ & $2(18,2 \%)$ & $8(32 \%)$ & $10(40 \%)$ & $7(28 \%)$ \\
\hline
\end{tabular}




\begin{tabular}{c|c|c|c|c|c|c}
\hline \multirow{2}{*}{ Alimentos } & \multicolumn{2}{|c|}{$\begin{array}{r}\text { Crianças de 2 a 10 anos incom- } \\
\text { pletos (n=11) }\end{array}$} & \multicolumn{3}{|c}{ Adolescentes (n=25) } \\
\cline { 2 - 7 } & Sim & Não & Não Sabe & Sim & Não & $\begin{array}{c}\text { Não } \\
\text { Sabe }\end{array}$ \\
\hline $\begin{array}{c}\text { Macarrão instantâ- } \\
\text { neo, salgadinhos de } \\
\text { pacote ou biscoitos } \\
\text { salgados }\end{array}$ & $1(9,1 \%)$ & $8(72,7 \%)$ & $2(18,2 \%)$ & $8(32 \%)$ & $10(40 \%)$ & $7(28 \%)$ \\
\hline $\begin{array}{c}\text { Biscoitos recheados, } \\
\text { doces ou gulosei- } \\
\text { mas (balas, pirulitos, } \\
\text { chicletes, caramelos, } \\
\text { gelatina) }\end{array}$ & $1(9,1 \%)$ & $9(81,8 \%)$ & $1(9,1 \%)$ & $7(28 \%)$ & $11(44 \%)$ & $7(28 \%)$ \\
\hline
\end{tabular}

Fonte: Dados da pesquisa, 2018.

Segundo Brasil (2012), atualmente há uma grande exposição dos escolares a esses alimentos, bem como o apelo publicitário com relação a esses produtos. $\mathrm{O}$ consumo de alimentos industrializados de alta densidade energética (com grande quantidade de gorduras e/ou açúcar) e de baixo valor nutricional (pobre em minerais e vitaminas), aliado ao comportamento sedentário, é apontado como a principal causa do aumento do excesso de peso nessa faixa etária.

O consumo excessivo de energia, proveniente da ingestão de produtos ultraprocessados, como estes que foram consumidos em excesso pelos adolescentes avaliados, não garantem uma adequada nutrição, podendo causar inclusive carências de micronutrientes, mesmo na presença da adequação calórica (SILVA et al. 2010).

Conforme Jesus (2017), durante a infância e a adolescência, são comuns hábitos alimentares errôneos com o consumo excessivo de bebidas adoçadas e produtos ultraprocessados, ricos em gordura, açúcar e sal, e o baixo consumo de legumes, verduras e frutas. Esses hábitos estão associados ao menor gasto energético diário, o que explica as tendências crescentes de sobrepeso e obesidade. 
A tabela 3 mostra a distribuição dos marcadores do consumo alimentar do SISVAN pelos adultos entrevistados de ambos os sexos. Mais de um terço consumiu hambúrguer/e ou embutidos, bebidas adoçadas, macarrão instantâneo, salgadinhos de pacote ou biscoitos salgados, biscoitos recheados, doces ou guloseimas. $\mathrm{O}$ consumo desses alimentos, conforme as orientações para avaliação dos marcadores de consumo alimentar para atenção básica (BRASIL, 2015a) devem ser desestimulados em todas as faixas etárias, pois apresentam quantidades elevadas de gordura saturada, trans, sódio, açúcar e baixo teor de nutrientes. Por outro lado, o consumo de feijão, frutas frescas, legumes e vegetais frescos variados deve ser recomendado, pelo fato destes serem ricos em minerais, vitaminas e fibras alimentares.

Tabela 3 - Distribuição dos marcadores do consumo alimentar do SISVAN por adultos beneficiários do Programa Bolsa Família de um município do sul catarinense

\begin{tabular}{c|c|c|c}
\hline \multirow{2}{*}{ Alimentos } & \multicolumn{3}{|c}{ Adultos (n=41) } \\
\cline { 2 - 4 } Feijão & Sim & Não & Não Sabe \\
\hline $\begin{array}{c}\text { Frutas frescas (não considerar suco } \\
\text { de frutas) }\end{array}$ & $16(39,0 \%)$ & $19(46,3 \%)$ & $6(14,6 \%)$ \\
\hline $\begin{array}{c}\text { Verduras e/ou legumes (não con- } \\
\text { siderar batata, mandioca, aipim, } \\
\text { macaxeira, cará e inhame) }\end{array}$ & $13(31,7 \%)$ & $23(56,1 \%)$ & $5(12,2 \%)$ \\
\hline $\begin{array}{c}\text { Hambúrguer e/ou embutidos (pre- } \\
\text { sunto, mortadela, salame, linguiça, } \\
\text { salsicha) }\end{array}$ & $13(31,7 \%)$ & $23(56,1 \%)$ & $5(12,2 \%)$ \\
\hline $\begin{array}{c}\text { Bebidas adoçadas (refrigerante, } \\
\text { suco de caixinha, suco em pó, água } \\
\text { de coco de caixinha, xaropes de } \\
\text { guaraná/groselha, suco de fruta com } \\
\text { adição de açúcar) }\end{array}$ & $13(31,7 \%)$ & $23(56,1 \%)$ & $5(12,2 \%)$ \\
\hline $\begin{array}{c}\text { Macarrão instantâneo, salgadinhos } \\
\text { de pacote ou biscoitos salgados }\end{array}$ & $15(36,6 \%)$ & $21(51,2 \%)$ & $5(12,2 \%)$ \\
\hline
\end{tabular}

Fonte: Dados da pesquisa, 2018. 
Conforme as orientações para avaliação dos marcadores de consumo alimentar para atenção básica (BRASIL, 2015a), o consumo de processados derivados de carne (embutidos, hambúrgueres, salsichas, salames, linguiças etc.) leva à absorção de quantidades elevadas de gordura saturada gordura, colesterol e sódio, devendo ser evitados, assim como o consumo de alimentos ricos em açúcares. $\mathrm{O}$ consumo de carnes brancas, vermelhas ou ovos, por possuírem alta qualidade de proteínas, vitaminas e minerais, deve ser estimulado, assim como a ingestão de frutas, legumes e vegetais frescos, por estes serem fontes de fibras e diversos micronutrientes e por apresentarem baixa quantidade de calorias.

As pesquisas realizadas em 2016 pela VIGITEL (BRASIL, 2017) mostram que apenas um a cada três adultos consomem frutas e hortaliças ao menos cinco dias da semana, sendo que destes o consumo é maior entre as mulheres. Em relação ao consumo de feijão, em 2012, o consumo regular era de 67,5\%, passando para 61,3\% em 2016.

Segundo o Guia Alimentar para a População Brasileira (BRASIL, 2014a), é preciso que os alimentos in natura ou minimamente processados sejam base para uma alimentação nutricionalmente balanceada, com variedade na oferta de produtos de origem vegetal, saborosos e aceitos culturalmente dentro de um sistema alimentar que garanta sustentabilidade para o ambiente. Entretanto, a constituição da autonomia para escolhas alimentares mais saudáveis não é exclusivamente uma decisão individual, pois está relacionada também com o ambiente vivido e com fatores econômicos, sociais e culturais que podem ter um impacto positivo ou negativo sobre o padrão alimentar das pessoas.

As crianças de 6 a 24 meses não se fizeram presentes na entrevista, portanto não foi possível realizar aferição de peso e estatura para classificação do estado nutricional. Havia um menino de 3 anos, cuja relação índica de massa corporal para idade (IMC/I) foi de em $19,13 \mathrm{~kg} / \mathrm{m}^{2}$, sendo este classifica acima do percentil $99(\mathrm{P}>99)$ e, portanto, como obesidade para a idade. A estatura para todas as fases, tanto de crianças, adolescentes e adultos estava adequada para a idade. Não houve casos de idosos. 
Neste estudo, verificou-se que das 7 crianças presentes, $74,10 \%$ estavam acima do peso, sendo $28,6 \%$ em situação de sobrepeso, $14,2 \%$ obesidade e $28,6 \%$ obesidade grave. Em relação aos adolescentes, $45,5 \%$ se encontraram acima do peso, do quais $27,3 \%$ estavam em sobrepeso. Em situação de obesidade e de obesidade grave chegou-se ao percentual de $9,1 \%$ respectivamente.

A obesidade é uma doença crônica, definida como o excesso de gordura corporal, que é causada por fatores de risco genéticos e ambientais (SOTELO; COLUGNATI; TADDEI, 2004). Conforme Abrantes, Lamounier e Colosimo (2002), a prevalência de obesidade está crescendo intensamente na infância e na adolescência e tende a persistir na vida adulta: cerca de 50\% das crianças obesas aos 6 meses de idade e de $80 \%$ das crianças obesas aos 5 anos de idade permanecerão obesas. Por isso é de suma importância a prevenção, o diagnóstico e o tratamento da obesidade na infância.

Segundo Enes e Slater (2010), a presença de obesidade na adolescência tem sido associada ao aparecimento precoce hipertensão arterial (HAS), dislipidemias, aumento da ocorrência de diabetes mellitus tipo dois (DM2), distúrbios na esfera emocional, o que pode comprometer a postura e causar problemas no aparelho locomotor. Os efeitos da obesidade precoce também serão vistos a longo prazo, aumentando o risco de mortalidade, especialmente por doença coronariana nos adultos que foram obesos na infância e na adolescência.

$\mathrm{Na}$ amostra de 40 indivíduos adultos de 20 a 60 anos incompletos deste estudo, apenas $25 \%$ das mulheres estão na faixa de peso adequada, sendo que $31,25 \%$ estão com sobrepeso e $40,55 \%$ estão com algum tipo de obesidade. No caso dos homens entrevistados, $62,5 \%$ estão com o peso adequado e $37,5 \%$ estão acima do peso.

Veloso e Silva (2010) analisaram uma amostra com 1005 adultos de 20 a 59 anos, sendo 612 mulheres e 393 homens, todos residentes no Maranhão. Nas mulheres, a prevalência de obesidade foi de $15,3 \%$, e de sobrepeso de $26,8 \%$. Nos homens, a prevalência de obesidade foi de $13,0 \%$ e de sobrepeso $31,1 \%$. No presente estudo, das 32 mulheres analisadas 31,2\% (10) apresentaram sobrepeso e $40,5 \%$ (13) obesidade. Dentre os homens, verificou-se $12,5 \%$ 
(1) de sobrepeso e $25,0 \%$ (2) de obesidade, achados que se diferenciam dos estudos acima, apesar da diferença amostral.

Linhares et al. (2012) realizaram um estudo com amostra de 2.448 indivíduos adultos em Pelotas-RS. A prevalência de obesidade foi de 21,7\% nos homens e 29,2\% nas mulheres. Já Holanda et al. (2011) avaliaram o estado nutricional de 464 adultos, entre 20 e 59 anos, residentes da zona urbana de Teresina-PI. As prevalências de sobrepeso e de obesidade foram, respectivamente, de $30 \%$ e $7,7 \%$.

Conforme os dados da VIGITEL 2016 (BRASIL, 2017), o excesso de peso em adultos cresceu $26,3 \%$ e a obesidade $60 \%$, de 2006 a 2016. Os dados mostram também que a prevalência de obesidade duplica a partir dos 25 anos e é mais frequente nos indivíduos com grau menor de escolaridade.

No presente estudo, das famílias entrevistadas, 55,9\% (19) estão em situação de Insegurança Alimentar Grave (IAG). Essa situação é alarmante, visto que apenas uma família vive em situação de segurança alimentar. Nos domicílios com menores de 18 anos, o percentual de IAG foi de 65,4\% (17). As informações podem ser verificadas na tabela 4 .

Tabela 4 - Situação de Segurança Alimentar e Nutricional nos domicílios dos beneficiários do Programa Bolsa Família de um município do sul catarinense

\begin{tabular}{c|c|c|c|c|c|c}
\hline \multirow{2}{*}{ Classificação } & \multicolumn{2}{c|}{$\begin{array}{c}\text { Domicílios com } \\
\text { menores de 18 anos }\end{array}$} & \multicolumn{2}{c|}{$\begin{array}{c}\text { Domicílios sem } \\
\text { menores de 18 anos }\end{array}$} & \multicolumn{2}{c}{$\begin{array}{c}\text { Total de } \\
\text { domicílios }\end{array}$} \\
\cline { 2 - 7 } & $\mathbf{N}$ & $\mathbf{\%}$ & $\mathbf{N}$ & $\mathbf{\%}$ & $\mathbf{n}$ & $\%$ \\
\hline Segurança Alimentar & 1 & 3,8 & - & - & 1 & 2,9 \\
\hline $\begin{array}{c}\text { Insegurança Alimentar } \\
\text { Leve }\end{array}$ & 6 & 23,0 & 3 & 37.5 & 9 & 26,5 \\
\hline $\begin{array}{c}\text { Insegurança Alimentar } \\
\text { Moderada }\end{array}$ & 2 & 7,7 & 3 & 37,5 & 5 & 14,7 \\
\hline $\begin{array}{c}\text { Insegurança Alimentar } \\
\text { Grave }\end{array}$ & 17 & 65,4 & 2 & 25,0 & 19 & 55,9 \\
\hline Total & 26 & 76,4 & 8 & 23,6 & 34 & 100 \\
\hline
\end{tabular}

Fonte: Dados da Pesquisa, 2018. 
Segundo as pesquisas feitas pelo IBASE em 2008, apesar do aumento no consumo de alimentos, uma parcela significativa dos beneficiários do PBF encontrava-se em situação de IAG (21\%, representando 2,3 milhões de famílias). Apesar do aumento declarado no consumo de alimentos, uma parcela significativa dos beneficiários (21\%, representando 2,3 milhões de famílias) encontra-se em situação de IAG (fome entre adultos e/ou crianças da família); outros 34\% (ou 3,8 milhões de famílias) estão em situação de insegurança alimentar moderada (IAM) (restrição na quantidade de alimentos na família) (INESC, 2008).

Apresentam insegurança alimentar leve (IAL), quando não há falta de alimentos, mas preocupação em relação ao consumo no futuro, 28\% (ou 3,1 milhões de famílias) e 17\% (ou 1,9 milhão de famílias), que estão em situação de segurança alimentar e nutricional.

Em Maceió-AL, no ano de 2011 (CABRAL et al., 2013), foram avaliadas 204 famílias, totalizando 847 beneficiários do PBF. Destes, 91,2\% apresentavam algum tipo de IA. Nos domicílios com crianças e adolescentes, todas as famílias apresentaram IA, sendo 20,3\% leve, 46,9\% moderada e 32,9\% grave. Nos domicílios só com adultos, 29,5\% estavam em situação de segurança alimentar, 32,8\% em IAL, 29,5\% IAM e 8,2\% IAG. Observou-se, nesse estudo, que as proporções de IA foram maiores nas classes econômicas $\mathrm{D}$ e nos domicílios com quatro ou mais moradores que não recebem outro benefício, com renda menor que $\mathrm{R} \$ 545,00$, sem moradia própria, com água proveniente de poço/ cacimbão, sem esgoto, cuja mãe era respondente, com idade menor que 40 anos, que não trabalhava e que tinha a circunferência de cintura inadequada $\mathrm{e}$ excesso de peso (CABRAL et al., 2013).

Também em 2011 foi traçado em Viçosa-MG o perfil socioeconômico e a IA das famílias beneficiárias do PBF que possuíam crianças com idade de dois a seis anos, de ambos os sexos, residentes na zona urbana (SPERANDIO; PRIORE, 2015). Participaram da pesquisa 243 famílias, das quais 72,8\% apresentaram alguma IA, sendo 47,3\% consideradas IAL, 10,7\% IAM e $14,8 \%$ IAG. A prevalência de IA foi mais elevada nos domicílios com menor nível 
socioeconômico, maior número de moradores, que possuíam água tratada por filtração e cujas mães tinham baixa escolaridade.

Monteiro, Souza e Pinho (2014) realizaram em Montes Claros-MG uma análise da IA e nutricional dos beneficiários do PBF. Foram entrevistados 72 beneficiários e obtidas informações sobre idade, renda, escolaridade situação de IA e nutricional, consumo alimentar, avaliação do serviço de saúde, conhecimentos em nutrição e estado nutricional. Foi adotada a EBIA com 15 questões que refletem a IA em diferentes níveis. De modo geral, todas as famílias apresentaram algum grau de IA, sendo 48,6\% IAL, 34,7\% IAM e 16,7\% IAG.

\section{CONCLUSÃO}

O estudo possibilitou uma análise do PBF e sua possível relação com a Insegurança Alimentar e Nutricional da população, mostrando a necessidade de Políticas Públicas voltadas para o combate à fome e à vulnerabilidade social, e também a efetivação de ações educativas por parte dos profissionais de saúde, especificamente nutricionistas, que favoreçam a adoção de hábitos alimentares mais saudáveis.

O elevado índice de sobrepeso e obesidade observado neste estudo, em ambos os sexos e nas diversas faixas etárias, pode estar relacionado com a alimentação pobre em vitaminas e minerais provenientes das leguminosas, hortaliças e frutas e ainda estar atribuído ao consumo de alimentos ultraprocessados que favorecem não apenas o ganho de peso, mas a carência de micronutrientes.

A alta prevalência de IA encontrada no estudo, principalmente a de grau mais severo, pode estar relacionada ao fato de a amostra ser composta exclusivamente por beneficiários do PBF, grupo que pelos critérios de inclusão do programa, são famílias em situação de extrema pobreza e vulnerabilidade social.

Para contribuir com a segurança alimentar e nutricional da população beneficiária do PBF, o profissional nutricionista pode realizar o diagnóstico 
nutricional da população estudada, bem como, avaliar a segurança alimentar e nutricional dessas famílias e atuar diretamente com os beneficiários promovendo ações de educação alimentar e nutricional ou promovendo capacitações para os demais profissionais da saúde básica.

\section{REFERÊNCIAS}

BARROS, D. C. de Avaliação nutricional antropométrica de gestantes adolescentes no município do Rio de Janeiro. 2009. 129 f. Tese (Doutorado em Ciências) - Fundação Oswaldo Cruz, Escola Nacional de Saúde Pública Sergio Arouca, Rio de Janeiro, 2009.

BRASIL. Ministério da Saúde. Saúde no Brasil: Contribuições para a Agenda de Prioridades de Pesquisa. Brasília: Ministério da Saúde, 2004b.

BRASIL. Ministério da Saúde. Guia alimentar para a população brasileira. 2. ed. Brasília, DF: Ministério da Saúde, Secretaria de Atenção à Saúde, Departamento de Atenção Básica, 2014a. 156 p.

BRASIL. Ministério do Desenvolvimento Social. Bolsa Família garante direitos e autonomia às mulheres. Publicado em 09 de março de 2016. Disponível em: <http://mds.gov.br/area-de-imprensa/noticias/2016/marco/ bolsa-familia-garante-direi tos-e-autonomia-as-mulheres $>$. Acesso em: 16 ago. 2018.

BRASIL. Ministério da Saúde. Orientações para avaliação de marcadores de consumo alimentar na atenção básica. Brasília: DF: Ministério da Saúde, Secretaria de Atenção à Saúde, Departamento de Atenção Básica, 2015a. 33 p.

BRASIL. Ministério da Saúde. Secretaria de Atenção à Saúde. Departamento de Atenção Básica. Saúde da criança: aleitamento materno e alimentação complementar. 2. ed. Brasília: Ministério da Saúde, 2015b. 184 p. 
BRASIL. Manual de Orientação para a alimentação escolar na Educação Infantil, Ensino Fundamental, Ensino Médio e na Educação de Jovens e Adultos. 2. ed. Brasília, DF: Ministério da Saúde, 2012. 48 p.

BRASIL. Vigitel Brasil 2016: vigilância de fatores de risco e proteção para doenças crônicas por inquérito telefônico: estimativas sobre frequência e distribuição sociodemográfica de fatores de risco e proteção para doenças crônicas em 2016. Brasília, DF: Ministério da Saúde, 2017. 159 p.

BRITO, J. G. de; COSTA, E. R. Titularidade Feminina no Programa Bolsa Família: Questões de Gênero e Segurança Alimentar. Revista Tropos: Comunicação, Sociedade e Cultura, v. 1, n. 3, n.p., 2015. Disponível em: $<$ http://revistas.ufac.br/revista/index.php/tropos/article/view/185>. Acesso em: 16 ago. 2018.

CABRAL, M. J. et al. Perfil socioeconômico, nutricional e de ingestão alimentar de beneficiários do Programa Bolsa Família. Revista Estud Av., São Paulo, v. 27, n. 78, p. 71-87, 2013.

CARVALHO, C. A. de et al. Consumo alimentar e adequação nutricional em crianças brasileiras: revisão sistemática. Revista Paul Pediatr., São Paulo, v. 33, n. 2, p. 211-221, jun. 2015.

CORADI, F. de B.; BOTTARO, S. M.; KIRSTEN, V. R. Consumo Alimentar de Crianças de Seis a Doze Meses e Perfil Sociodemográfico Materno. Revista Demetra: Alimentação, Nutrição \& Saúde, Arvorezinha, v. 12, n. 3, p. 733750, 26 ago. 2017.

CORREAA, A. B. F.; MARTINS, G. de S. W. Estado nutricional de escolares em escolas públicas, de uma cidade de médio porte e pequeno porte, no sul de Santa Catarina. 2018. 57 f. Trabalho de Conclusão de Curso (Graduação em Nutrição) - Universidade do Extremo Sul Catarinense, Criciúma, 2018.

CUSTÓDIO, M. B.; YUBA, T. Y; CYRILlO, D. C. Política de segurança alimentar e nutricional no Brasil: uma análise da alocação de recursos. Revista Panam Salud Publica, v. 33, n. 2, p. 144-50, 2013. 
DIAS, M. C. A. P.; FREIRE, L. M. S.; FRANCESCHINI, S. do C. C. Recomendações para alimentação complementar de crianças menores de dois anos. Revista Nutr., Campinas, v. 23, n. 3, p. 475-486, jun. 2010.

ENES, C. C.; SLATER, B. Obesidade na adolescência e seus principais fatores determinantes. Revista Bras Epidemiol. v. 13, n. 1, São Paulo, mar. 2010.

FIATES, G. M. R.; AMBONI, R. D. de M. C.; TEIXEIRA, E. Comportamento consumidor, hábitos alimentares e consumo de televisão por escolares de Florianópolis. Revista Nutr. Campinas, v. 21, n. 1, p. 105-114, fev. 2008.

INSTITUTO DE ESTUDOS SOCIOECONÔMICOS - INESC. O perfil do Bolsa Família. Publicado em 2 de julho de 2008. Disponível em: <http://www. inesc.org.br/noticias/noticias-gerais/2008/julho/o-perfil-do-bolsa-familia $>$. Acesso em: 12 fev. 2018.

JESUS, L. S. de. Consumo alimentar de adolescentes de uma escola no município de Itabaiana-Sergipe. 2017. Monografia (Graduação em Nutrição) - Universidade Federal de Sergipe, Lagarto, 2017.

LINHARES, R. et al. Distribuição de obesidade geral e abdominal em adultos de uma cidade no Sul do Brasil. Cad Saúde Pública. Rio de Janeiro, v. 28, n. 3, mar. 2012.

MONTEIRO, F. O Programa Bolsa Família no contexto da segurança alimentar e nutricional no Brasil. Revista Sau \& Transf Soc. Florianópolis, v. 5, n. 3, p. 91-97, 2014.

MONTEIRO, E. L. F.; SOUZA, C. R.; PINHO, L. Diagnóstico de Insegurança Alimentar e Nutricional entre beneficiários do Programa Bolsa Família participantes de oficina de intervenção nutricional. Revista Segurança Alimentar e Nutricional. Campinas, v. 21, n. 2, p. 469-480, 2014. Disponível em: <file://C:/Users/usuario/Downloads/8634476-3457-1-SM.pdf>. Acesso: 18 ago. 2017.

MOREIRA, F. D.; CARRASCHI, K. R. P. Avaliação do Consumo Alimentar de Crianças Menores de 5 anos em Criciúma. 2017.44 f. Trabalho de Conclusão de 
Curso (Graduação em Nutrição) - Universidade do Extremo Sul Catarinense, Criciúma, 2017.

PEREZ, A. I. de la C. et al. Monitoramento do estado nutricional de usuários de Unidades Básicas de Saúde no Estado de São Paulo por meio do Sistema de Vigilância Alimentar e Nutricional (SISVAN). Revista BEPA, São Paulo, v. 10, n. 116, ago. 2013.

ROSA, J. A. O. da. Estado nutricional e consumo de alimentos de beneficiários do Programa Bolsa Família em uma Unidade Básica de Saúde de Porto Alegre - RS. 2011. 68- f. TCC (Graduação) Curso de Nutrição - Universidade Federal do Rio Grande do Sul, Porto Alegre, 2011.

SEGALL-CORREA, A. M.; MARIN-LEON, L. A. Segurança alimentar no Brasil: proposição e usos da Escala Brasileira de Medida da Insegurança Alimentar (EBIA) de 2003 a 2009. Revista Segurança Alimentar e Nutricional. Campinas, v. 16, n. 2, p. 1-19, 2009.

SILVA, J. V. L. da et al. Consumo alimentar de crianças e adolescentes residentes em uma área de invasão em Maceió, Alagoas, Brasil. Revista Bras Epidemiol. v. 13, n. 1, p. 83-93, 2010.

SISTEMA DE VIGILÂNCIA ALIMENTAR E NUTRICIONAL - SISVAN. Formulário de Marcadores de Consumo Alimentar. Elaborado em 2015. Disponível em: <http://dabsistemas.saude.gov.br/sistemas/sisvanV2/public/ file/ficha_marcadores_alimentar.pdf $>$. Acesso em: 14 mar. 2018.

SOTELO, Y. de O. M.; COLUGNATI, F. A. B.; TADDEI, J. A. de A. C. Prevalência de sobrepeso e obesidade entre escolares da rede pública segundo três critérios de diagnóstico antropométrico. Cad Saúde Pública. Rio de Janeiro, v. 20, n. 1, p. 233-240, fev. 2004.

SPERANDIO, N.; PRIORE, S. E. Prevalência de insegurança alimentar domiciliar e fatores associados em famílias com pré-escolares, beneficiárias do Programa Bolsa Família em Viçosa, Minas Gerais, Brasil. Revista Epidemiol Serv Saúde. Brasília, v. 24, n. 4, p. 739-748, out./dez. 2015. 
TOLONI, M. H. de A. et al. Introdução de alimentos industrializados e de alimentos de uso tradicional na dieta de crianças de creches públicas no município de São Paulo. Revista Nutr. v. 24, n.1, p .61-70, 2011

VELOSO, H. J. F.; SILVA, A. A. M. da. Prevalência e fatores associados à obesidade abdominal e ao excesso de peso em adultos maranhenses. Revista Bras Epidemiol. São Paulo, v. 13, n. 3, p. 400-412, set. 2010.

ZVEIBRÜCKER, F. P.; MIRAGLIA, F. Avaliação do consumo alimentar de pré-escolares frequentadores de EMEIs no município de Nova Santa Rita-RS. CIPPUS - Revista de Iniciação Científica do Unilasalle. v. 1, n. 1, maio 2012. 